\title{
Joint role of non-HDL cholesterol and glycated haemoglobin in predicting future coronary heart disease events among women with type 2 diabetes
}

\author{
M. B. Schulze ${ }^{1,7}$ • I. Shai ${ }^{1,2,3}$ • J. E. Manson ${ }^{2,4,5}$ • T. $\mathbf{L i}^{1}$ • N. Rifai ${ }^{6}$ R. Jiang1, 2 F. B. Hu ${ }^{1,2,5}$ \\ ${ }^{1}$ Department of Nutrition, Harvard School of Public Health, Boston, Massachusetts, USA \\ 2 Department of Epidemiology, Harvard School of Public Health, Boston, Massachusetts, USA \\ ${ }^{3}$ S. Daniel Abraham International Center for Health and Nutrition, Department of Epidemiology, Ben-Gurion University, \\ Beer-Sheva, Israel \\ ${ }^{4}$ Division of Preventive Medicine, Department of Medicine, Brigham and Women's Hospital and Harvard Medical School, \\ Boston, Massachusetts, USA \\ 5 Channing Laboratory, Department of Medicine, Brigham and Women's Hospital and Harvard Medical School, Boston, \\ Massachusetts, USA \\ ${ }^{6}$ Department of Laboratory Medicine, Children's Hospital and Harvard Medical School, Boston, Massachusetts, USA \\ ${ }^{7}$ Department of Epidemiology, German Institute of Human Nutrition, Potsdam-Rehbruecke, Nuthetal, Germany
}

\begin{abstract}
Aims/hypothesis. Non-HDL cholesterol (the sum of LDL, VLDL and IDL cholesterol) is considered to be particularly valuable in the management of dyslipidaemia in type 2 diabetes. However, it remains uncertain whether the association between non-HDL cholesterol and cardiovascular risk in type 2 diabetes depends on the status of hyperglycaemia. We aimed to determine whether non-HDL cholesterol predicts CHD events among diabetic women independently of currently established risk factors and the status of glycaemic control.

Methods. We prospectively followed 921 diabetic women in the Nurses' Health Study, who were free of cardiovascular disease at the time that blood was drawn in 1989/90. During 10 years of follow-up, we identified 122 incident CHD cases.

Results. After adjustment for age, BMI, smoking, alcohol consumption, and other lifestyle risk factors, the multivariate relative risks (RRs) of CHD for extreme quartiles were 1.97 (95\% CI: 1.14-3.43) for non-HDL
\end{abstract}

cholesterol, 1.78 (1.02-3.11) for apolipoprotein B100 , and 1.93 (1.15-3.22) for LDL cholesterol. However, the association between non-HDL cholesterol and CHD risk was only apparent among women with elevated fasting triglycerides (RR for extreme quartiles: $3.80 ; p=0.045) . \mathrm{HbA}_{1} \mathrm{c}$ was strongly associated with increased CHD risk (RR for increase by 1 unit: 1.24; 95\% CI: 1.13-1.35), and both non-HDL cholesterol and $\mathrm{HbA}_{1} \mathrm{c}$ additively predicted CHD risk (RR for the combination of high non-HDL cholesterol and high $\mathrm{HbA}_{1} \mathrm{c}$ [tertiles]: 4.59).

Conclusions/interpretation. Our study suggests that non-HDL cholesterol and $\mathrm{HbA}_{1} \mathrm{c}$ are potent predictors of CHD risk in diabetic women. Therapies to lower CHD risk in diabetic patients should emphasise both glycaemic control and lipid lowering.

Keywords $\mathrm{CHD} \cdot \mathrm{HbA}_{1} \mathrm{c} \cdot$ Hypertriglyceridaemia Lipoproteins - LDL cholesterol - Non-HDL cholesterol · Prospective studies · Risk factors · Type 2 diabetes . Women
Received: 15 May 2004 / Accepted: 18 July 2004

Published online: 15 December 2004

(C) Springer-Verlag 2004

M. B. Schulze (『)

Department of Epidemiology,

German Institute of Human Nutrition, Potsdam-Rehbruecke, Arthur-Scheunert-Allee 114-116, 14558 Nuthetal, Germany

E-mail: mschulze@mail.dife.de

\section{Introduction}

LDL is widely considered to be the major atherogenic lipoprotein and the primary target of lipid-lowering therapy [1]. In the majority of people who have low

Abbreviations: apoB $_{100}$, apolipoprotein B-100 .

$\mathrm{CABG}$, coronary bypass surgery $\cdot \mathrm{CVD}$, cardiovascular disease $\cdot$ MI, myocardial infarction $\cdot$ PTCA, coronary angioplasty $\cdot \mathrm{RR}$, relative risks 
triglyceride levels, LDL contains the bulk of atherogenic cholesterol. However, triglyceride-rich lipoproteins VLDL and IDL contribute to the total of atherogenic cholesterol, particularly in subjects with elevated triglycerides. As a consequence, non-HDL cholesterol, the sum of LDL, VLDL and IDL cholesterol, as a measure of total atherogenic cholesterol, has been identified as a secondary target of lipid therapy among subjects with elevated triglycerides by the Adult Treatment Panel III of the National Cholesterol Education Program [1]. Because the most common pattern of dyslipidaemia in patients with type 2 diabetes is elevated triglyceride levels and decreased HDL cholesterol levels, with LDL cholesterol not being significantly different from that in non-diabetic individuals [2], non-HDL cholesterol has been considered to be particularly valuable in the management of dyslipidaemia in type 2 diabetes [3]. However, it remains uncertain whether the association between non-HDL cholesterol and cardiovascular disease (CVD) risk in type 2 diabetes depends on the status of hyperglycaemia. Given the relatively scarce data at hand, we conducted this study to determine whether non-HDL cholesterol predicts CHD events among diabetic women independently of currently established risk factors and the status of glycaemic control.

\section{Subjects and methods}

Subjects. The Nurses' Health Study is a prospective cohort study of 121,700 US nurses aged 30 to 55 years at study initiation in 1976. This cohort is followed through biennial mailed questionnaires focusing on various lifestyle factors and health outcomes. In addition, in 1989/90 32,826 study participants provided blood samples by overnight courier. Among participants who returned blood samples, 1,194 had a confirmed diagnosis of type 2 diabetes (as reported on a validated supplementary questionnaire sent to all women who reported a diagnosis of diabetes) at baseline or during follow-up through 1996. The present study included 921 women who did not report a diagnosis of myocardial infarction (MI), coronary bypass surgery $(\mathrm{CABG})$, coronary angioplasty (PTCA) or stroke on any of the biennial questionnaires before blood collection, and for whom complete biomarker data were available. The study was approved by the Human Research Committees at the Harvard School of Public Health and the Brigham and Women's Hospital; completion of the self-administered questionnaire was considered to imply informed consent.

Diabetes and cardiovascular endpoint definitions. Cases of diabetes were reported by the respondent on the biennial questionnaires. We mailed a supplementary questionnaire to all women reporting a diagnosis of diabetes to obtain details about the type of diabetes (type 1 or 2), date of diagnosis, symptoms, diagnostic tests and hypoglycaemic treatment. In accordance with the criteria of the National Diabetes Data Group [4], confirmation of type 2 diabetes required at least one of the following self-reports on the supplementary questionnaire: (i) an elevated plasma glucose concentration (fasting plasma glucose $\geq 7.8 \mathrm{mmol} / 1$, random plasma glucose $\geq 11.1 \mathrm{mmol} / 1$, and/or plasma glucose $\geq 11.1 \mathrm{mmol} / \mathrm{l}$ after $\geq 2 \mathrm{~h}$ during an OGTT) plus at least one classic symptom (excessive thirst, polyuria, weight loss or hunger); (ii) no symptoms, but at least two elevated plasma glucose concentrations (according to the above criteria) on different occasions; or (iii) treatment with hypoglycaemic medication (insulin or oral hypoglycaemic agent). We used the National Diabetes Data Group criteria to define type 2 diabetes because our subjects were diagnosed prior to the release of the American Diabetes Association criteria of 1997 [5]. The validity of self-reported diagnosis of type 2 diabetes by supplementary questionnaire has been established by a separate validation study through medical record reviews. Of the 84 women, 71 provided permission for medical record review and medical records were obtained for 62 . The diagnosis of type 2 diabetes was confirmed in 61 of these women (98.4\%) [6].

CHD endpoints consisted of fatal CHD, non-fatal MI, and CABG/PTCA. The endpoint did not include angina pectoris. Non-fatal MI was confirmed by reviewing medical records using the criteria of the World Health Organization of symptoms, plus either typical electrocardiographic changes or elevated cardiac enzyme levels [7]. Physicians who reviewed the records had no knowledge of the self-reported risk factor status. Deaths were reported by next of kin, the postal system, and through records of the National Death Index. Using all sources combined it is estimated that follow-up for deaths was over $98 \%$ complete [8]. Fatal MI and coronary disease were confirmed by review of medical records or autopsy reports with the permission of the next of kin. The cause listed on the death certificate was not sufficient to confirm a coronary death. Sudden deaths (i.e. death within $1 \mathrm{~h}$ of symptom onset in a man without known disease that could explain death) were included in the fatal CHD category.

Blood collection and processing. Blood was collected from 32,826 participants in 1989-1990. Interested participants were sent a blood collection kit that included supplies (blood tubes, tourniquet, needles, bandage, coolant pack) and instructions. Participants arranged for the blood to be drawn and sent the samples back by prepaid overnight courier. Most samples arrived within $24 \mathrm{~h}$ after the blood was drawn. After arrival in the lab, samples were centrifuged and aliquoted into cryotubes as plasma, buffy coat, and erythrocytes. Cryotubes were stored in liquid nitrogen freezers at a temperature of $-130^{\circ} \mathrm{C}$ or lower.

All biomarker analyses were carried out in the laboratory of N. Rifai, which is certified by the NHLBI/CDC lipid standardisation program. The concentrations of total cholesterol, HDL cholesterol, and triglycerides were simultaneously measured on the Hitachi 911 analyser using reagents and calibrates from Roche Diagnostics (Indianapolis, Ind., USA); coefficients of variation for these measurements were below 1.8\%. LDL cholesterol concentrations were measured by a homogenous direct method from Genzyme Corporation (Cambridge, Mass., USA). The day-to-day variability at LDL cholesterol concentrations of $2.33,2.74$ and $3.34 \mathrm{mmol} / \mathrm{l}$ was below $3.1 \%$. Apolipoprotein B-100 $\left(\operatorname{apoB}_{100}\right)$ concentrations were measured via an immunonephelometric assay using reagents and calibrators from Wako Chemicals USA (Richmond, Va., USA) with a day-today variability of less than $5 \%$. $\mathrm{HbA}_{1} \mathrm{c}$ concentrations were determined based on turbidimetric immunoinhibition using haemolysed whole blood or packed red cells. The day-to-day variability at $\mathrm{HbA}_{1} \mathrm{c}$ concentrations of 5.5 and $9.1 \%$ was 1.9 and $3.0 \%$ respectively. We calculated non-HDL cholesterol as the difference between total and HDL cholesterol.

Assessment of lifestyle exposures. Participants provided information biennially on their age, weight, smoking status, aspirin use, postmenopausal hormone use, history of high blood pressure, insulin use, oral hypoglycaemic medication, cholesterol- 
Table 1. Baseline characteristics of cases and non-cases

\begin{tabular}{|c|c|c|c|}
\hline & $\begin{array}{l}\text { Diabetic women } \\
\text { with incident CHD } \\
(n=122)\end{array}$ & $\begin{array}{l}\text { Diabetic women } \\
\text { without CHD events } \\
(n=799)\end{array}$ & $p$ value \\
\hline Weight (kg) & $77.8 \pm 21.2$ & $78.3 \pm 22.8$ & 0.841 \\
\hline $\operatorname{BMI}\left(\mathrm{kg} / \mathrm{m}^{2}\right)^{\mathrm{a}}$ & $29.7 \pm 5.9$ & $30.1 \pm 6.4$ & 0.951 \\
\hline Physical activity (METs/week) & $19.3 \pm 90.4$ & $32.1 \pm 134.7$ & 0.152 \\
\hline Postmenopausal hormone use (\%) & 31.2 & 26.4 & 0.272 \\
\hline Insulin use $(\%)$ & 29.5 & 17.7 & 0.002 \\
\hline Oral hypoglycaemic medication (\%) & 32.8 & 18.3 & $<0.001$ \\
\hline Cholesterol-lowering drug use $(\%)$ & 7.4 & 3.4 & 0.034 \\
\hline Parental history of CHD (\%) & 30.3 & 21.7 & 0.033 \\
\hline History of hypertension (\%) & 72.1 & 58.0 & 0.003 \\
\hline LDL cholesterol (mmol/l) & $3.79 \pm 0.94$ & $3.58 \pm 0.97$ & 0.011 \\
\hline HDL cholesterol (mmol/l) & $1.29 \pm 0.41$ & $1.34 \pm 0.38$ & 0.090 \\
\hline Non-HDL cholesterol (mmol/l) & $4.85 \pm 1.11$ & $4.47 \pm 1.13$ & $<0.001$ \\
\hline Ratio of total cholesterol : HDL cholesterol & $5.22 \pm 2.10$ & $4.64 \pm 1.48$ & 0.001 \\
\hline Fasting triglycerides $(\mathrm{mmol} / \mathrm{l})^{\mathrm{c}}$ & $2.56 \pm 1.62$ & $2.15 \pm 1.60$ & 0.009 \\
\hline $\operatorname{ApoB}_{100}(\mathrm{~g} / \mathrm{l})$ & $1.09 \pm 0.24$ & $1.02 \pm 0.25$ & 0.001 \\
\hline Lipoprotein (a) ( $\mu \mathrm{mol} / \mathrm{l})$ & $0.74 \pm 0.90$ & $0.63 \pm 0.89$ & 0.142 \\
\hline $\mathrm{HbA}_{1} \mathrm{c}(\%)$ & $7.6 \pm 1.9$ & $6.8 \pm 1.7$ & $<0.001$ \\
\hline
\end{tabular}

Data are means \pm SD or percentages; tests were two-sided Wilcoxon rank test or chi square test. a 119 cases and 772 non-cases due to missing values; b 118 cases and 752 non-cases due to missing values; c 94 cases and 526 non-cases

lowering drug use, and physical activity. If the weight was missing we used the weight reported on the preceding questionnaire instead. We calculated BMI as the ratio of weight (in $\mathrm{kg}$ ) to squared height (in $\mathrm{m}^{2}$ ), the latter being assessed in 1976. Physical activity was computed as metabolic equivalents per week using the duration per week of various forms of exercise, weighting each activity by its intensity level [9]. History of high blood pressure was determined from self-reports preceding the blood collection. Parental history of coronary heart disease was reported in 1976. Alcohol intake was estimated with a dietary questionnaire in 1990, 1994 and 1998.

Statistical analysis. We used Cox proportional hazards analysis stratified on 5-year age categories and over each 2-year followup interval to estimate the relative risks (RRs) for each biomarker quartile compared with the lowest quartile. Person-months of follow-up accumulated starting in June 1990. Participants who were diagnosed with CHD, stroke or died during follow-up were censored at the date of diagnosis or death. All other participants were followed through June 2000. Tests for trend were calculated by assigning the median values to increasing categories of biomarkers. Multivariate models included the following covariates: physical activity (quintiles), alcohol intake (0, 0.1-4.9, 5.0-14.9, $\geq 15.0 \mathrm{~g} /$ day, missing), parental history of $\mathrm{CHD}$, history of high blood pressure at time of blood being drawn, aspirin use (<1/week, 1-2/week, 3-6/week, 7-14/week, $\geq 15 /$ week, missing), smoking (never, past, current $1-14$, current $15-24$, current $\geq 25$ cigarettes/day), postmenopausal hormone use (premenopausal, never, past, current, missing), and BMI (missing, <23, 23-24, 25-27, 28-29, 30-34, $\geq 35$ ). We tested for effect modification by triglyceride levels, aspirin use, insulin use and oral hypoglycaemic medication by performing Cox proportional hazards analyses stratified by these variables and by evaluating interaction terms. In addition, we evaluated whether lipid markers were associated with risk independently of the status of glycaemic control by crossclassifying these markers with $\mathrm{HbA}_{1}$ c levels and by evaluating interaction terms, which were computed by modelling the tertile medians as continuous variables. While we included in our main analysis participants who reported a diagnosis of diabetes after blood collection $(n=245)$ and who reported the use of cholesterol-lowering medication at baseline $(n=36)$ to enhance the statistical power of our study, the severity of insulin resistance and the use of cholesterol-lowering medication may affect blood lipid values and their metabolic consequences. To evaluate whether these inclusions affected our results, we repeated the analysis excluding these women. A $p$ value of less than 0.05 was considered statistically significant. All statistical analyses were performed using SAS statistical software Version 8.0 (SAS institute, Cary, N.C., USA).

\section{Results}

During an average of 7.4 years of follow-up $(6,835$ person-years), we identified 122 incident cases of CHD (46 non-fatal MI, 21 fatal MI, 55 CABG/ PTCA), confirmed by medical records. Among the study population of 921 women, cases (women with subsequent CHD events) had a significantly higher baseline age, used insulin, oral hypoglycaemic medication and cholesterol-lowering medication more fre- 
Table 2. Relative risks (95\% CIs) of CHD according to quartiles of biomarkers among 921 diabetic women

\begin{tabular}{|c|c|c|c|c|c|}
\hline & \multicolumn{4}{|c|}{ Quartiles of biomarker } & \multirow[t]{2}{*}{$p$ for trend } \\
\hline & 1 (low) & 2 & 3 & 4 (high) & \\
\hline \multicolumn{6}{|l|}{ Non-HDL cholesterol } \\
\hline Median (mmol/l) & 3.28 & 4.11 & 4.77 & 5.81 & \\
\hline Age-adjusted RR & 1.00 & $1.51(0.84-2.70)$ & $1.49(0.84-2.64)$ & $2.12(1.23-3.63)$ & 0.007 \\
\hline Multivariate-adjusted RR ${ }^{\mathrm{a}}$ & 1.00 & $1.46(0.81-2.64)$ & $1.53(0.85-2.73)$ & $1.97(1.14-3.43)$ & 0.016 \\
\hline \multicolumn{6}{|l|}{$\mathrm{ApoB}_{100}$} \\
\hline Median (g/l) & 0.74 & 0.93 & 1.10 & 1.31 & \\
\hline Age-adjusted RR & 1.00 & $1.23(0.68-2.24)$ & $1.68(0.96-2.93)$ & $1.97(1.14-3.39)$ & 0.007 \\
\hline Multivariate-adjusted RR ${ }^{\mathrm{a}}$ & 1.00 & $1.17(0.64-2.14)$ & $1.70(0.97-3.00)$ & $1.78(1.02-3.11)$ & 0.020 \\
\hline \multicolumn{6}{|l|}{ Total cholesterol } \\
\hline Median (mmol/l) & 4.62 & 5.46 & 6.10 & 7.09 & \\
\hline Age-adjusted RR & 1.00 & $1.04(0.59-1.83)$ & $1.10(0.63-1.91)$ & $1.75(1.06-2.90)$ & 0.016 \\
\hline Multivariate-adjusted RR ${ }^{\mathrm{a}}$ & 1.00 & $1.08(0.61-1.91)$ & $1.14(0.65-2.01)$ & $1.77(1.06-2.95)$ & 0.016 \\
\hline \multicolumn{6}{|l|}{ LDL cholesterol } \\
\hline Median (mmol/l) & 2.54 & 3.31 & 3.87 & 4.65 & \\
\hline Age-adjusted RR & 1.00 & $1.25(0.72-2.18)$ & $1.06(0.60-1.87)$ & $1.81(1.09-3.00)$ & 0.025 \\
\hline Multivariate-adjusted RR ${ }^{\mathrm{a}}$ & 1.00 & $1.34(0.77-2.35)$ & $1.11(0.63-1.98)$ & $1.93(1.15-3.22)$ & 0.016 \\
\hline \multicolumn{6}{|l|}{ HDL cholesterol } \\
\hline Median (mmol/l) & 0.94 & 1.17 & 1.38 & 1.77 & \\
\hline Age-adjusted RR & 1.00 & $0.58(0.36-0.94)$ & $0.41(0.24-0.70)$ & $0.70(0.44-1.10)$ & 0.126 \\
\hline Multivariate-adjusted RR ${ }^{\mathrm{a}}$ & 1.00 & $0.66(0.40-1.07)$ & $0.44(0.25-0.76)$ & $0.85(0.52-1.39)$ & 0.391 \\
\hline \multicolumn{6}{|l|}{ Fasting triglycerides ${ }^{b}$} \\
\hline Median (mmol/l) & 0.98 & 1.56 & 2.25 & 3.45 & \\
\hline Age-adjusted RR & 1.00 & $0.84(0.45-1.56)$ & $0.90(0.49-1.64)$ & $1.64(0.94-2.83)$ & 0.027 \\
\hline Multivariate-adjusted RR ${ }^{\mathrm{a}}$ & 1.00 & $0.70(0.37-1.34)$ & $0.80(0.42-1.49)$ & $1.42(0.79-2.54)$ & 0.070 \\
\hline \multicolumn{6}{|c|}{ Total cholesterol: HDL cholesterol ratio } \\
\hline Median & 3.13 & 3.99 & 4.99 & 6.33 & \\
\hline Age-adjusted RR & 1.00 & $0.60(0.32-1.12)$ & $1.37(0.83-2.27)$ & $1.56(0.95-2.54)$ & 0.006 \\
\hline Multivariate-adjusted $\mathrm{RR}^{\mathrm{a}}$ & 1.00 & $0.55(0.29-1.05)$ & $1.27(0.74-2.15)$ & $1.36(0.80-2.32)$ & 0.028 \\
\hline \multicolumn{6}{|l|}{ Lipoprotein (a) } \\
\hline Median ( $\mu \mathrm{mol} / 1)$ & 0.06 & 0.19 & 0.38 & 1.76 & \\
\hline Age-adjusted RR & 1.00 & $0.85(0.50-1.44)$ & $1.00(0.60-1.66)$ & $1.26(0.78-2.03)$ & 0.158 \\
\hline Multivariate-adjusted RR ${ }^{\mathrm{a}}$ & 1.00 & $0.99(0.57-1.70)$ & $1.11(0.66-1.87)$ & $1.42(0.86-2.33)$ & 0.106 \\
\hline \multicolumn{6}{|l|}{$\mathrm{HbA}_{1} \mathrm{c}$} \\
\hline Median (\%) & 5.21 & 5.80 & 6.90 & 8.97 & \\
\hline Age-adjusted RR & 1.00 & $2.56(1.24-5.31)$ & $3.11(1.53-6.31)$ & $4.66(2.36-9.20)$ & $<.001$ \\
\hline Multivariate-adjusted RR ${ }^{a}$ & 1.00 & $2.49(1.19-5.23)$ & $3.19(1.56-6.53)$ & $4.92(2.46-9.85)$ & $<.001$ \\
\hline
\end{tabular}

a Adjusted for age, physical activity (quintiles), alcohol intake (0, 0.1-4.9, 5.0-14.9, $\geq 15.0 \mathrm{~g} / \mathrm{day}$, missing), parental history of CHD, history of high blood pressure at blood collection, aspirin use (<1/week, 1-2/week, 3-6/week, 7-14/week, $\geq 15$.0/week, missing), smoking (never, past, current $1-14$, cur-

quently, and had higher levels of total cholesterol, LDL cholesterol, non-HDL cholesterol, fasting triglycerides, apoB $\mathrm{B}_{100}, \mathrm{HbA}_{1} \mathrm{c}$, and a higher total cholesterol : HDL cholesterol ratio (Table 1). In addition, cases were more likely to have a history of hypertension, a history of angina, and a parental history of rent 15-24, current $\geq 25$ cigarettes/day), postmenopausal hormone use (premenopausal, never, past, current, missing), and BMI (missing, <23, 23-24, 25-27, 28-29, 30-34, $\geq 35$ ); b 94 cases and 526 non-cases

CHD at the time of blood collection. Although HDL cholesterol appeared to be lower in cases than in controls, this association was not statistically significant.

Increasing levels of non-HDL cholesterol, apoB ${ }_{100}$, total cholesterol and LDL cholesterol were strongly associated with progressively higher risk of CHD (Ta- 
Table 3. Relative risks ${ }^{\mathrm{a}}$ (95\% CIs) of cardiovascular events according to levels of non-HDL by triglyceride levels, aspirin use, insulin use and oral hypoglycaemic drug use among 921 diabetic women

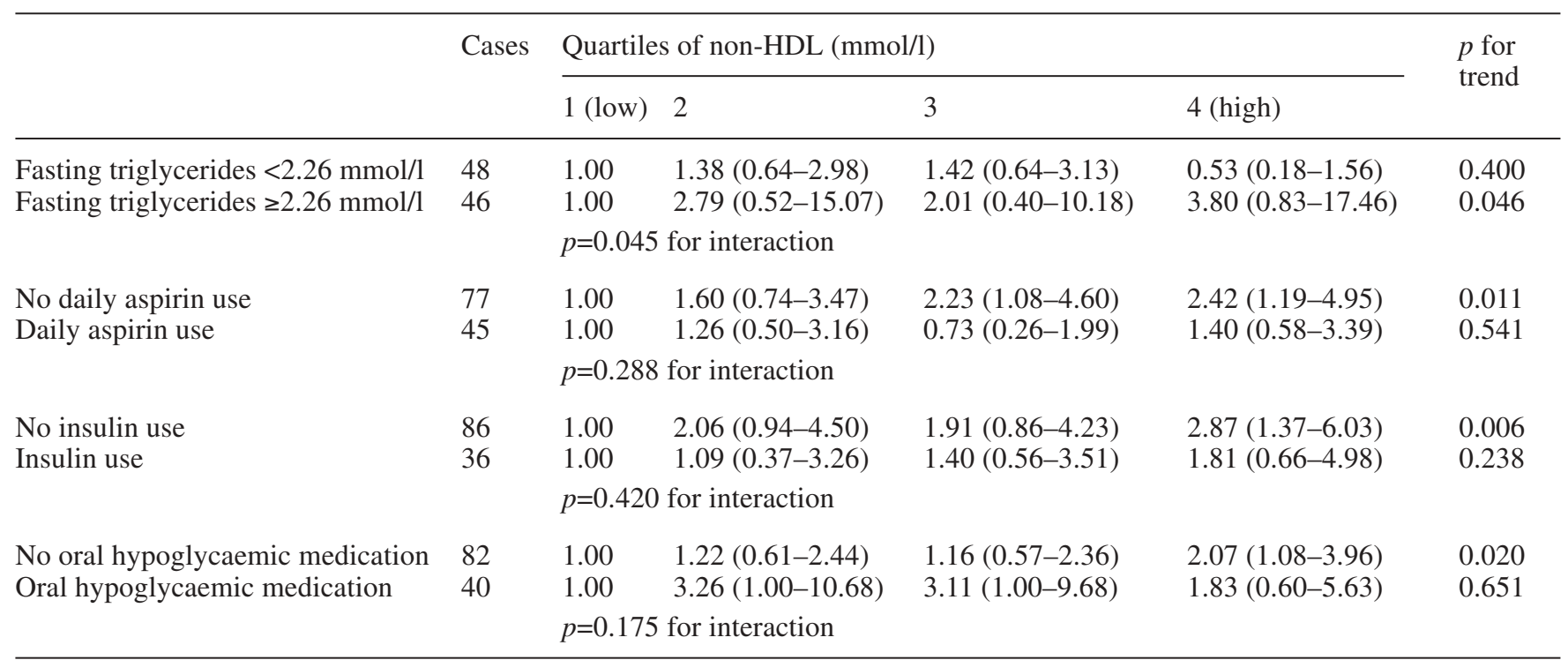

a Relative risks adjusted for age, physical activity (quartiles), alcohol intake $(0,0.1-4.9, \geq 5.0 \mathrm{~g} /$ day $)$, parental history of CHD, history of high blood pressure at blood draw, smoking (never, past, current), postmenopausal hormone use (premeno- pausal or never, past, current), and BMI (missing, <23, 23-24, 25-27, 28-29, $\geq 30$ ) and aspirin use (non-daily, daily) depending on model ble 2). The age-adjusted RRs for extreme quartiles were 2.12 for non-HDL cholesterol, 1.97 for $\mathrm{apoB}_{100}, 1.81$ for LDL cholesterol and 1.75 for total cholesterol. In addition, fasting triglycerides and an increased total cholesterol : HDL cholesterol ratio were associated with a moderate increase in risk (RRs for extreme quartiles: 1.64 and $1.56, p=0.027$ and $p=0.006$ respectively). HDL cholesterol was not significantly associated with risk. These associations remained similar after further adjustment for BMI, alcohol consumption, smoking, parental history of CHD, physical activity, history of high blood pressure, aspirin use, and postmenopausal hormone use, except for fasting triglycerides, where the positive association was no longer statistically significant $(p=0.070)$. In addition, $\mathrm{HbA}_{1} \mathrm{c}$ was strongly associated with increased CHD risk. The multivariate adjusted RR for an $\mathrm{HbA}_{1} \mathrm{c}$ increase of 1 unit was $1.24(95 \%$ CI: 1.13-1.35). Across increasing quartiles of $\mathrm{HbA}_{1} \mathrm{c}$, the RRs were 1.00, 2.49, 3.19 and $4.92(p<0.001)$.

In a secondary analysis, we excluded 245 participants who were diagnosed with type 2 diabetes after blood collection ( $n=106$ for remaining CHD cases). Non-HDL cholesterol remained strongly associated with risk (multivariate-adjusted RR for extreme quartiles: $1.84 ; 95 \%$ CI: $1.03-3.28 ; p=0.037$ ). In addition, exclusion of 36 women reporting use of cholesterollowering drugs at baseline did not materially change our results (multivariate-adjusted RR for extreme quartiles of non-HDL cholesterol: $1.79,95 \% \mathrm{CI}$ : $1.01-3.17, p=0.055)$.

We also evaluated whether the association between non-HDL cholesterol and CHD risk is modified by as- pirin use, oral hypoglycaemic medication, or the level of fasting triglycerides (Table 3). Tests for interactions were all non-significant for aspirin use, insulin use and oral hypoglycaemic medication. However, the association appeared to be stronger (RR for extreme quartiles: $3.80 ; p=0.046)$ among women with high triglyceride levels $(\geq 2.26 \mathrm{mmol} / \mathrm{l})$ compared with among women with low triglyceride levels $(p=0.045)$.

We examined the joint effect of blood lipids (LDL cholesterol, non-HDL cholesterol, HDL cholesterol, apoB ${ }_{100}$, and fasting triglycerides) and $\mathrm{HbA}_{1} \mathrm{c}$ by cross-classifying participants according to their lipids and their $\mathrm{HbA}_{1} \mathrm{c}$ (Fig. 1). $\mathrm{HbA}_{1} \mathrm{c}$ appeared to be associated with an increased CHD risk at any lipid level. Also, the associations of non-HDL cholesterol, LDL cholesterol and $\mathrm{apoB}_{100}$ with $\mathrm{CHD}$ risk were largely independent of $\mathrm{HbA}_{1} \mathrm{c}$ levels. The RRs for the combination of high lipid level (upper tertile) and high $\mathrm{HbA}_{1} \mathrm{c}$ level (upper tertile) compared with the opposite extreme were 4.59 for non-HDL cholesterol, 4.14 for LDL cholesterol and 4.78 for $\mathrm{apoB}_{100}$. Higher HDL cholesterol appeared to be associated with a decreased risk among women with low $\mathrm{HbA}_{1} \mathrm{c}$ (RR for extreme tertiles: 0.39), but this association was weaker or not existent among women with moderate or high $\mathrm{HbA}_{1} \mathrm{c}$. However, the test for interaction was not statistically significant $(p=0.124)$. Fasting triglycerides were associated with an increased risk among women with low $\mathrm{HbA}_{1} \mathrm{c}$ (RR for extreme tertiles: 3.32), but this association was not present among women with high $\mathrm{HbA}_{1} \mathrm{c}$. This interaction was statistically significant $(p=0.033)$. 

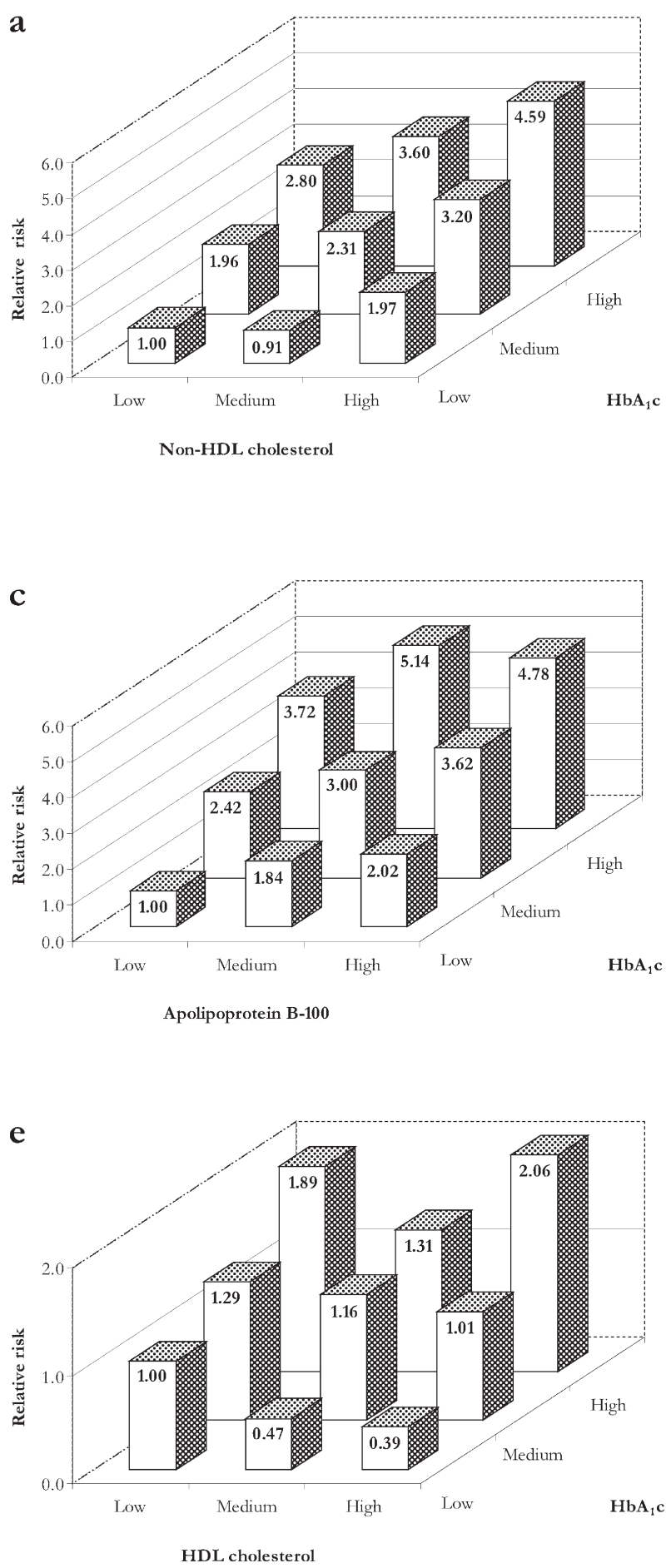

Fig. 1. Relative risk of $\mathrm{CHD}$ by different levels (tertiles) of $\mathrm{HbA}_{1} \mathrm{c}$ and non-HDL cholesterol (a), LDL cholesterol (b), apoB $_{100}(\mathbf{c})$, fasting triglycerides (d) and HDL cholesterol (e)

\section{Discussion}

In this prospective cohort of 921 diabetic women without a previous history of cardiovascular disease at the beginning of this study, higher levels of non-HDL cholesterol, apoB ${ }_{100}$ and LDL cholesterol at baseline
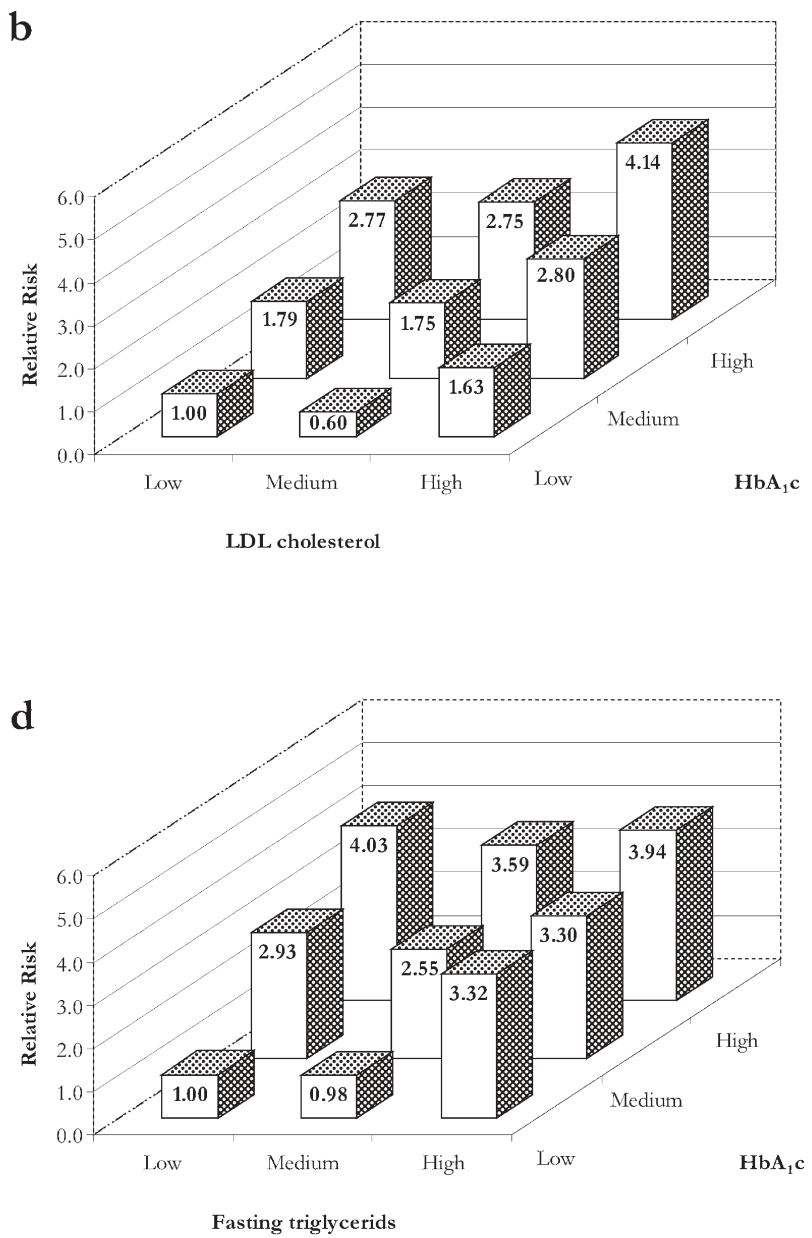

were associated with a significantly increased future risk of CHD independently of age, BMI, smoking and other lifestyle CHD risk factors. These associations were not modified by the status of glycaemic control. Non-HDL cholesterol appeared to be a strong predictor of CHD risk; however, the association with CHD risk may be stronger among women with elevated triglycerides ( $\geq 2.26 \mathrm{mmol} / \mathrm{l})$. HDL cholesterol was not a significant predictor of CHD risk, and the total cholesterol : HDL cholesterol ratio was associated with only a moderately increased risk.

The role of non-HDL cholesterol as a predictor of CVD risk among subjects with type 2 diabetes has been evaluated in two previous studies $[10,11]$. The RR comparing extreme tertiles of non-HDL cholesterol was 1.80 (95\% CI: 1.32-2.46) in the Strong Heart Study, a study among 772 American Indian men and 1336 women [10]. Similarly, a high non-HDL cholesterol $(\geq 5.2 \mathrm{mmol} / \mathrm{l})$ was associated with a $60 \%$ increase in CHD mortality and a $70 \%$ increase of all CHD events in a study among 581 Finnish men and 478 women [11]. Both studies suggested that nonHDL cholesterol is a stronger predictor of CHD than LDL cholesterol, although this was not formally tested. Similarly, non-HDL cholesterol predicted risk of CVD among 779 subjects with impaired glucose me- 
tabolism or diagnosed type 2 diabetes in the Hoorn Study [12]. Studies in non-diabetic subjects generally found strong associations between non-HDL cholesterol and CVD risk [13, 14, 15, 16, 17].

In our study, $\mathrm{HbA}_{1} \mathrm{c}$ was the most potent predictor of CHD risk, but non-HDL cholesterol, LDL cholesterol and $\mathrm{apoB}_{100}$ were associated with increased CHD risk independently of the status of glycaemic control. These results suggests that both tight glycaemic control and lipid lowering are needed to reduce the excess risk of CHD in patients with type 2 diabetes [18]. Similar to our study, HDL cholesterol and the total cholesterol : HDL cholesterol ratio were not associated or only moderately associated with CVD risk among diabetic women in the Strong Heart Study [10]. However, in our study, an inverse association between HDL cholesterol and CHD risk appeared to be present among women with low $\mathrm{HbA}_{1} \mathrm{c}$ but not among women with high $\mathrm{HbA}_{1} \mathrm{c}$. Likewise, fasting triglyceride levels were only associated with an increased CHD risk among women with low $\mathrm{HbA}_{1}$ c. One potential explanation is that the effects of HDL cholesterol and triglycerides are masked by poor glycaemic control. However, our findings are in contrast to the study by Letho et al., who found no significant interactions between fasting glucose levels and HDL cholesterol and triglyceride levels [11].

In contrast to our study, non-HDL cholesterol was associated with risk among diabetic subjects independently of their triglyceride levels in the Strong Heart Study [10]. This observation has led to the opinion that non-HDL cholesterol may be generally superior to LDL cholesterol as a therapy target in type 2 diabetes [3]. However, our results suggest that non-HDL cholesterol may predict CHD risk only among women with elevated triglyceride levels. In most persons with triglyceride levels below $2.26 \mathrm{mmol} / \mathrm{l}$, VLDL cholesterol is not substantially elevated [19] and non-HDL cholesterol correlates highly with LDL cholesterol $[20,21]$. Therefore, at lower triglyceride levels, nonHDL cholesterol would be expected to provide little additional power to predict CHD. When triglyceride levels are $2.26 \mathrm{mmol} / \mathrm{l}$ or higher, VLDL cholesterol levels are distinctly raised [19] and LDL cholesterol concentrations are less well correlated with non-HDL cholesterol levels $[20,21]$. In the presence of high serum triglycerides, non-HDL cholesterol, therefore, will better represent the concentrations of all atherogenic lipoproteins than will LDL cholesterol alone [1]. Our finding supports the recommendation of the Adult Treatment Panel III to use non-HDL cholesterol as a secondary target of therapy (after LDL cholesterol lowering) among subjects with elevated triglycerides [1]. Also, non-HDL cholesterol determination appears to be easier and more reliable than LDL cholesterol determination in diabetic subjects (LDL cholesterol is usually calculated requiring fasting triglyceride levels below $4.52 \mathrm{mmol} / \mathrm{l}$, in contrast to the direct measurement in our study) [3], and might therefore be superior to LDL cholesterol as a target for management of dyslipidaemia in type 2 diabetes.

One limitation of our study is the relatively small sample size, which may have led to unstable estimates in stratified analyses, particularly for fasting samples. In addition, we used one blood sample per subject only and were therefore not able to account for intraindividual variation of lipoproteins. This may have led to an underestimation of the effect size, particularly for triglycerides. A further limitation of our study is the reliance on self-reported body weight. It is possible that underreporting of body weight, particularly among heavier women, may have led to an underestimation of BMI. However, correlation between self-reported and technician-measured body weight was found to be high in this cohort ( $r=0.96)$ [22]. Body weight was missing for $3 \%$ of women at baseline and for up to $10 \%$ during each follow-up cycle. We carried forward body weight from previous questionnaires if it was missing, to reduce misclassification, although this ignored potential weight gain during follow-up.

In conclusion, our results suggest that non-HDL cholesterol, LDL cholesterol and apoB $_{100}$ are strong predictors of CHD among diabetic women independently of the status of glycaemic control. Non-HDL cholesterol might be particularly valuable as a risk marker among diabetic women with elevated triglyceride levels. Both tight glycaemic control and lipid lowering are needed to reduce the excess risk of CHD in patients with type 2 diabetes.

Acknowledgements. This study was supported by research grants (CA87969, HL65582, HL34594 and DK58845) from the National Institutes of Health. M.B. Schulze was supported by a fellowship within the Postdoc-Program of the German Academic Exchange Service (DAAD). I. Shai was supported by the Fulbright Foundation (USA) and by the S. Daniel Abraham International Center for Health and Nutrition (Ben-Gurion University of the Negev, Israel). F.B. Hu is the recipient of an American Heart Association Established Investigator Award. The funding organisations had no role in the design and conduct of the study, in the collection, analysis and interpretation of the data, and in the preparation, review or approval of the manuscript.

\section{References}

1. Expert Panel on Detection, Evaluation, And Treatment of High Blood Cholesterol In Adults (Adult Treatment Panel III) (2001) Executive Summary of The Third Report of The National Cholesterol Education Program (NCEP), Expert Panel on Detection, Evaluation, And Treatment of High Blood Cholesterol In Adults (Adult Treatment Panel III). JAMA 285:2486-2497

2. Haffner SM (2004) Dyslipidemia management in adults with diabetes. Diabetes Care 27 [Suppl 1]:S68-S71

3. Hsia SH (2003) Non-HDL cholesterol: into the spotlight. Diabetes Care 26:240-242

4. National Diabetes Data Group (1979) Classification and diagnosis of diabetes mellitus and other categories of glucose intolerance. Diabetes 28:1039-1057 
5. Expert Committee on the Diagnosis and Classification of Diabetes Mellitus (1997) Report of the Expert Committee on the Diagnosis and Classification of Diabetes Mellitus. Diabetes Care 20:1183-1197

6. Colditz GA, Martin P, Stampfer MJ et al. (1986) Validation of questionnaire information on risk factors and disease outcomes in a prospective cohort study of women. Am J Epidemiol 123:894-900

7. Rose GA, Blackburn H, Gillum RF, Prineas RJ (1982) Cardiovascular survey methods. Monograph Series No. 56, WHO, Geneva

8. Stampfer MJ, Willett WC, Speizer FE et al. (1984) Test of the National Death Index. Am J Epidemiol 119:837-839

9. Wolf AM, Hunter DJ, Colditz GA et al. (1994) Reproducibility and validity of a self-administered physical activity questionnaire. Int J Epidemiol 23:991-999

10. Lu W, Resnick HE, Jablonski KA et al. (2003) Non-HDL cholesterol as a predictor of cardiovascular disease in type 2 diabetes: the strong heart study. Diabetes Care 26:16-23

11. Lehto S, Ronnemaa T, Haffner SM, Pyorala K, Kallio V, Laakso M (1997) Dyslipidemia and hyperglycemia predict coronary heart disease events in middle-aged patients with NIDDM. Diabetes 46:1354-1359

12. Bos G, Dekker JM, Nijpels G et al. (2003) A combination of high concentrations of serum triglyceride and non-highdensity-lipoprotein-cholesterol is a risk factor for cardiovascular disease in subjects with abnormal glucose metabolism-The Hoorn Study. Diabetologia 46:910-916

13. Menotti A, Spagnolo A, Scanga M, Dima F (1992) Multivariate prediction of coronary deaths in a 10 year follow-up of an Italian occupational male cohort. Acta Cardiol 47:311-320
14. Frost PH, Davis BR, Burlando AJ et al. (1996) Serum lipids and incidence of coronary heart disease. Findings from the Systolic Hypertension in the Elderly Program (SHEP). Circulation 94:2381-2388

15. Gardner CD, Fortmann SP, Krauss RM (1996) Association of small low-density lipoprotein particles with the incidence of coronary artery disease in men and women. JAMA 276:875-881

16. Cui Y, Blumenthal RS, Flaws JA et al. (2001) Non-highdensity lipoprotein cholesterol level as a predictor of cardiovascular disease mortality. Arch Intern Med 161:14131419

17. Nishizawa Y, Shoji T, Kakiya R et al. (2003) Non-highdensity lipoprotein cholesterol (non-HDL-C) as a predictor of cardiovascular mortality in patients with end-stage renal disease. Kidney Int [Suppl]:S117-S120

18. Haffner SJ, Cassells H (2003) Hyperglycemia as a cardiovascular risk factor. Am J Med 115 [Suppl 8A]:6S-11S

19. The Lipid Research Clinics Program Epidemiology Committee (1979) Plasma lipid distributions in selected North American populations: the Lipid Research Clinics Program Prevalence Study. Circulation 60:427-439

20. Vega GL, Grundy SM (1990) Does measurement of apolipoprotein B have a place in cholesterol management? Arteriosclerosis 10:668-671

21. Abate N, Vega GL, Grundy SM (1993) Variability in cholesterol content and physical properties of lipoproteins containing apolipoprotein B-100. Atherosclerosis 104:159-171

22. Willett W, Stampfer MJ, Bain C et al. (1983) Cigarette smoking, relative weight, and menopause. Am J Epidemiol 117:651-658 